\title{
Commutators of cycles in permutation groups
}

\author{
Aleš Vavpetič * \\ Fakulteta za Matematiko in Fiziko, Univerza v Ljubljani, \\ Jadranska ulica 19, SI-1111 Ljubljana, Slovenija
}

Received 10 January 2013, accepted 26 September 2014, published online 27 May 2015

\begin{abstract}
We prove that for $n \geq 5$, every element of the alternating group $A_{n}$ is a commutator of two cycles of $A_{n}$. Moreover we prove that for $n \geq 2$, a $(2 n+1)$-cycle of the permutation group $S_{2 n+1}$ is a commutator of a $p$-cycle and a $q$-cycle of $S_{2 n+1}$ if and only if the following three conditions are satisfied (i) $n+1 \leq p, q$, (ii) $2 n+1 \geq p$, $q$, (iii) $p+q \geq 3 n+1$.
\end{abstract}

Keywords: Commutator, cycle, permutation, alternating group.

Math. Subj. Class.: $20 B 05$

\section{Introduction}

In 1951 O. Ore [9] conjectured that in a finite simple non-abelian group every element is a commutator. In the same paper he proved that the conjecture holds for the alternating group $A_{n}$, where $n \geq 5$, but the result had already been proved by G. A. Miller half a century earlier [7]. After Ore published the paper there were many papers devoted to the Ore conjecture: R. C. Thompson proved the Ore conjecture for the projective special linear groups $P S L_{n}(q)$ [10], [11], [12], R. Gow proved it for the projective simplectic groups $P S p_{2 n}(q)$, where $q \equiv 1(\bmod 4)$ [4], O. Bonten for the exceptional groups of Lie type of low rank [2], J. Neubüser, H. Pahlings, E. Cleuvers proved it for the sporadic groups [8], E. W. Ellers, N. Gordeev handled the finite simple groups of Lie type over a finite field $\mathbb{F}_{q}$, whenever $q \geq 9, \ldots$ M. W. Liebeck, E. A. O'Brien, A. Shalev, P. H. Tiep proved the Ore conjecture for the remaining cases [6] and the conjecture became the theorem. We refer the reader to the survey paper [5] for more historical notes about commutators and the Ore conjecture.

In this paper we prove a stronger version of the Ore conjecture for the simple alternating group $A_{n}$. In Section 2 it is shown that, for $n \geq 5$, every permutation of $A_{n}$ is actually a

* This research was supported by the Slovenian Research Agency grants P1-0292-0101 and J1-5435-0101.

E-mail address: ales.vavpetic@fmf.uni-lj.si (Aleš Vavpetič) 
commutator of two cycles of $A_{n}$. In particular, every even permutation of the symmetric group $S_{n}$ is a product of two conjugate cycles. Namely, if $\rho=[\sigma, \tau]=\sigma^{-1} \tau^{-1} \sigma \tau$, then $\rho$ is a product of $\sigma^{-1} \tau^{-1} \sigma$ and $\tau$ (and also a product of $\sigma^{-1}$ and $\tau^{-1} \sigma \tau$ ). Note that permutations $\tau$ and $\tau^{-1}$ are conjugate in $S_{n}$. In [1] it is proved that a $(2 n+1)$-cycle of $A_{2 n+1}$ is a product of two conjugate $l$-cycles of $A_{2 n+1}$ if and only if $l \geq n+1$. Hence this is a necessary condition for the existence of two $l$-cycles $\sigma$ and $\tau$ such that $[\sigma, \tau]$ is a $(2 n+1)$-cycle. In Section 3 it is shown that this is far from being a sufficient condition. More precisely, it is shown that, for $n \geq 2$, a $(2 n+1)$-cycle of $A_{2 n+1}$ is a commutator of a $p$-cycle and a $q$-cycle of $S_{2 n+1}$ if and only if $n+1 \leq p, q$ and $p+q \geq 3 n+1$. In particular, a $(2 n+1)$-cycle of $A_{2 n+1}(n \geq 2)$ is a commutator of $l$-cycles of $S_{2 n+1}$ if and only if $l \geq \frac{3 n+1}{2}$.

The image of an element $a$ under a permutation $\sigma$ is denoted by $a^{\sigma}$. Permutations are executed from left to right. The support $\operatorname{supp} \sigma$ of a permutation $\sigma$ is the set of all elements which are not fixed by $\sigma$.

Let $\sigma$ be a permutation, $a \in \operatorname{supp} \sigma$ and $x_{1}, \ldots, x_{n} \notin \operatorname{supp} \sigma$. We define permutations $\varphi\left(\sigma ; a, x_{1}, \ldots, x_{n}\right)$ and $\varepsilon(\sigma ; a)$ by

$$
t^{\varphi\left(\sigma ; a, x_{1}, \ldots, x_{n}\right)}= \begin{cases}x_{1}, & t=a, \\ x_{i+1}, & t \in\left\{x_{1}, \ldots, x_{n-1}\right\}, \\ a^{\sigma}, & t=x_{n}, \\ t^{\sigma}, & t \notin\left\{a, x_{1}, \ldots, x_{n}\right\},\end{cases}
$$

and

$$
t^{\varepsilon(\sigma ; a)}= \begin{cases}a, & t=a, \\ a^{\sigma}, & t=a^{\sigma^{-1}}, \\ t^{\sigma}, & t \notin\left\{a, a^{\sigma^{-1}}\right\} .\end{cases}
$$

If $\sigma$ is the $k$-cycle $\left(a_{1}, \ldots, a_{k}\right)$, then $\varphi\left(\sigma ; a_{k}, x_{1}, \ldots, x_{n}\right)$ is the $(k+n)$-cycle $\left(a_{1}, \ldots, a_{k}\right.$, $\left.x_{1}, \ldots, x_{n}\right)$ and $\varepsilon\left(\sigma ; a_{k}\right)$ is the $(k-1)$-cycle $\left(a_{1}, \ldots, a_{k-1}\right)$.

Let $\sigma$ and $\tau$ be permutations such that $\operatorname{supp} \sigma \cap \operatorname{supp} \tau=\emptyset$. For $a \in \operatorname{supp} \sigma$ and $b \in \operatorname{supp} \tau$, let $\psi(\sigma, \tau ; a, b)$ denote the permutation defined by

$$
t^{\psi(\sigma, \tau ; a, b)}= \begin{cases}t^{\sigma}, & t \in \operatorname{supp} \sigma-\{a\} \\ b^{\tau}, & t=a \\ t^{\tau}, & t \in \operatorname{supp} \tau-\{b\} \\ a^{\sigma}, & t=b .\end{cases}
$$

If $\tau$ is a $k$-cycle then $\psi(\sigma, \tau ; a, b)=\varphi\left(\sigma ; a, b^{\tau}, b^{\tau^{2}}, \ldots, b^{\tau^{k}}\right)$, and if $\sigma$ is a $k$-cycle then $\psi(\sigma, \tau ; a, b)=\varphi\left(\tau ; b, a^{\sigma}, a^{\sigma^{2}}, \ldots, a^{\sigma^{k}}\right)$.

\section{Permutations as commutators of cycles}

The proof that every permutation of $A_{n}(n \geq 5)$ is a commutator of two cycles is based on induction on the number and the lengths of cycles in the cycle decomposition of the permutation. In the following lemmas we describe how the application of $\varphi, \psi$, and $\varepsilon$ modify commutators. 
Lemma 2.1. Let $\sigma, \tau$ be permutations, $x \in \operatorname{supp} \sigma, y \in \operatorname{supp} \tau$, and $(\operatorname{supp} \sigma \cup \operatorname{supp} \tau) \cap$ $\left(\left\{x_{1}, \ldots, x_{n}\right\} \cup\left\{y_{1}, \ldots, y_{m}\right\}\right)=\emptyset$. Then for $t \notin\left\{x^{\sigma}, x^{\tau \sigma}, y^{\tau \sigma}, y^{\sigma^{-1} \tau \sigma}, x_{1}, \ldots, x_{n}\right.$, $\left.y_{1}, \ldots, y_{m}\right\}$ we have $t^{[\sigma, \tau]}=t^{\left[\varphi\left(\sigma ; x, x_{1}, \ldots, x_{n}\right), \varphi\left(\tau ; y, y_{1}, \ldots, y_{m}\right)\right]}$.

Proof. Denote $\tilde{\sigma}=\varphi\left(\sigma ; x, x_{1}, \ldots, x_{n}\right)$ and $\widetilde{\tau}=\varphi\left(\tau ; y, y_{1}, \ldots, y_{m}\right)$. For $t \notin\left\{x^{\sigma}, x^{\tau \sigma}\right.$, $\left.y^{\tau \sigma}, y^{\sigma^{-1} \tau \sigma}, x_{1}, \ldots, x_{n}, y_{1}, \ldots, y_{m}\right\}$ we have $t^{\sigma^{-1}}=t^{\widetilde{\sigma}^{-1}}$. Since $t \notin\left\{y^{\tau \sigma}, y_{1}, \ldots, y_{m}\right\}$, also $t^{\sigma^{-1}} \notin\left\{y^{\tau}, y_{1}, \ldots, y_{m}\right\}$ and therefore $t^{\sigma^{-1} \tau^{-1}}=t^{\widetilde{\sigma}^{-1} \widetilde{\tau}^{-1}}$. Since $t^{\sigma^{-1} \tau^{-1}} \notin\{x$, $\left.x_{1}, \ldots, x_{n}\right\}$ we have $t^{\sigma^{-1} \tau^{-1} \sigma}=t^{\widetilde{\sigma}^{-1} \widetilde{\tau}^{-1} \widetilde{\sigma}}$. And finally $t^{\sigma^{-1} \tau^{-1} \sigma} \notin\left\{y, y_{1}, \ldots, y_{m}\right\}$, hence $t^{[\sigma, \tau]}=t^{[\widetilde{\sigma}, \widetilde{\tau}]}$.

We record the following immediate consequence.

Corollary 2.2. Let $\sigma, \tau$ be permutations. Suppose that $a, b \in \operatorname{supp} \sigma$ such that $a^{\sigma}=$ $a^{\tau}=b$, and $(\operatorname{supp} \sigma \cup \operatorname{supp} \tau) \cap\left(\left\{x_{1}, \ldots, x_{n}\right\} \cup\left\{y_{1}, \ldots, y_{m}\right\}\right)=\emptyset$. Then for $t \notin$ $\left\{b^{\sigma}, b^{\tau \sigma}, x_{1}, \ldots, x_{n}, y_{1}, \ldots, y_{m}\right\}$ we have $t^{[\sigma, \tau]}=t^{\left[\varphi\left(\sigma ; b, x_{1}, \ldots, x_{n}\right), \varphi\left(\tau ; b, y_{1}, \ldots, y_{m}\right)\right]}$.

Lemma 2.3. Let $\sigma, \tau$ be permutations and $a, b \in \operatorname{supp} \sigma$ such that $b=a^{\sigma}=a^{\tau}$ and $c, d \notin \operatorname{supp} \sigma \cup \operatorname{supp} \tau$. Then

$$
[\varphi(\sigma ; b, c, d), \varphi(\tau ; b, d, c)]=\varphi\left([\sigma, \tau] ; b^{\tau \sigma}, c, d\right) .
$$

Proof. Denote $\widetilde{\sigma}=\varphi(\sigma ; b, c, d)$ and $\widetilde{\tau}=\varphi(\tau ; b, d, c)$. By Corollary 2.2, we have $t^{[\widetilde{\sigma}, \widetilde{\tau}]}=$ $t^{[\sigma, \tau]}$ for $t \notin\left\{b^{\sigma}, b^{\tau \sigma}, c, d\right\}$. Because

$$
\begin{aligned}
\left(b^{\tau \sigma}\right)^{[\widetilde{\sigma}, \widetilde{\tau}]} & =\left(b^{\tau}\right)^{\widetilde{\tau}-1} \widetilde{\sigma} \widetilde{\tau}=c^{\widetilde{\sigma} \widetilde{\tau}}=d^{\widetilde{\tau}}=c, \\
c^{[\widetilde{\sigma}, \widetilde{\tau}]} & =b^{\widetilde{\tau}^{-1} \widetilde{\sigma} \widetilde{\tau}}=a^{\widetilde{\sigma} \widetilde{\tau}}=b^{\widetilde{\tau}}=d, \\
d^{[\widetilde{\sigma}, \widetilde{\tau}]} & =c^{\widetilde{\tau}^{-1} \widetilde{\sigma} \widetilde{\tau}}=d^{\widetilde{\sigma} \widetilde{\tau}}=\left(b^{\sigma}\right)^{\widetilde{\tau}}=b^{\sigma \tau}=\left(b^{\tau \sigma}\right)^{[\sigma, \tau]}, \\
\left(b^{\sigma}\right)^{[\widetilde{\sigma}, \widetilde{\tau}]} & =d^{\widetilde{\tau}^{-1} \widetilde{\sigma} \widetilde{\tau}}=b^{\widetilde{\sigma} \widetilde{\tau}}=c^{\widetilde{\tau}}=b^{\tau}=\left(a^{\sigma}\right)^{\tau}=\left(b^{\tau^{-1}}\right)^{\sigma \tau}=\left(b^{\sigma}\right)^{[\sigma, \tau]},
\end{aligned}
$$

we have $[\varphi(\sigma ; b, c, d), \varphi(\tau ; b, d, c)]=\varphi\left([\sigma, \tau] ; b^{\tau \sigma}, c, d\right)$.

Lemma 2.4. Let $\sigma, \tau$ be permutations and $a, b \in \operatorname{supp} \sigma$ such that $b=a^{\sigma}=a^{\tau}$ and $c, d \notin \operatorname{supp} \sigma \cup \operatorname{supp} \tau$. Then

$$
\begin{aligned}
& {[\varphi(\sigma ; b, c, d), \varphi(\tau ; b, d)]=\varphi\left([\sigma, \tau] ; b^{\sigma}, c, d\right),} \\
& {[\varphi(\sigma ; b, d), \varphi(\tau ; b, c, d)]=\varphi\left([\sigma, \tau] ; b^{\sigma}, d, c\right) .}
\end{aligned}
$$

Proof. Denote $\widetilde{\sigma}=\varphi(\sigma ; b, c, d)$ and $\widetilde{\tau}=\varphi(\tau ; b, d)$. By Corollary 2.2, we have $t^{[\widetilde{\sigma}, \widetilde{\tau}]}=$ $t^{[\sigma, \tau]}$ for $t \notin\left\{b^{\sigma}, b^{\tau \sigma}, c, d\right\}$. Because

$$
\begin{aligned}
\left(b^{\sigma}\right)^{[\widetilde{\sigma}, \widetilde{\tau}]} & =d^{\widetilde{\tau}^{-1} \widetilde{\sigma} \widetilde{\tau}}=b^{\widetilde{\sigma} \widetilde{\tau}}=c^{\widetilde{\tau}}=c, \\
c^{[\widetilde{\sigma}, \widetilde{\tau}]} & =b^{\widetilde{\tau}^{-1} \widetilde{\sigma} \widetilde{\tau}}=a^{\widetilde{\sigma} \widetilde{\tau}}=b^{\widetilde{\tau}}=d, \\
d^{[\widetilde{\sigma}, \widetilde{\tau}]} & =c^{\widetilde{\tau}^{-1} \widetilde{\sigma} \widetilde{\tau}}=c^{\widetilde{\sigma} \widetilde{\tau}}=d^{\widetilde{\tau}}=b^{\tau}=\left(a^{\sigma}\right)^{\tau}=\left(b^{\tau^{-1}}\right)^{\sigma \tau}=\left(b^{\sigma}\right)^{[\sigma, \tau]}, \\
\left(b^{\tau \sigma}\right)^{[\widetilde{\sigma}, \widetilde{\tau}]} & =\left(b^{\tau}\right)^{\widetilde{\tau}^{-1} \widetilde{\sigma} \widetilde{\tau}}=d^{\widetilde{\sigma} \widetilde{\tau}}=\left(b^{\sigma}\right)^{\widetilde{\tau}}=b^{\sigma \tau}=\left(b^{\tau \sigma}\right)^{[\sigma, \tau]},
\end{aligned}
$$

we have $[\varphi(\sigma ; b, c, d), \varphi(\tau ; b, d)]=\varphi\left([\sigma, \tau] ; b^{\sigma}, c, d\right)$. 
Because $[\sigma, \tau]^{-1}=[\tau, \sigma]$ and $\left(b^{\tau}\right)^{[\tau, \sigma]}=b^{\sigma}$, we have

$$
\begin{aligned}
{[\varphi(\sigma ; b, d), \varphi(\tau ; b, c, d)] } & =([\varphi(\tau ; b, c, d), \varphi(\sigma ; b, d)])^{-1}= \\
& =\varphi\left([\tau, \sigma] ; b^{\tau}, c, d\right)^{-1}= \\
& =\varphi\left([\sigma, \tau] ; b^{\sigma}, d, c\right) .
\end{aligned}
$$

Corollary 2.5. Let $\rho$ be $a(2 n+1)$-cycle and $n \geq 2$. For $p, q \in \mathbb{N}$ such that $p, q \leq 2 n+1$ and $p+q \geq 3 n+2$, there exist a p-cycle $\sigma$, a q-cycle $\tau$, and $a \in \operatorname{supp} \sigma$ such that $[\sigma, \tau]=\rho$, supp $\rho=\operatorname{supp} \sigma \cup \operatorname{supp} \tau$, and $a^{\sigma}=a^{\tau}$. In the case $q \neq 2 n+1$ we arrange that $a^{\sigma \sigma} \notin \operatorname{supp} \tau$.

Proof. If $n=2$ and $p \geq q$ then $(p, q) \in\{(5,5),(5,4),(5,3),(4,4)\}$ and we have

$$
\begin{aligned}
\left(a_{1}, a_{2}, a_{3}, a_{4}, a_{5}\right) & =\left[\left(a_{1}, a_{4}, a_{2}, a_{3}, a_{5}\right),\left(a_{1}, a_{4}, a_{3}, a_{5}, a_{2}\right)\right]= \\
& =\left[\left(a_{1}, a_{4}, a_{2}, a_{5}, a_{3}\right),\left(a_{1}, a_{4}, a_{3}, a_{5}\right)\right]= \\
& =\left[\left(a_{1}, a_{2}, a_{4}, a_{5}, a_{3}\right),\left(a_{1}, a_{2}, a_{5}\right)\right]= \\
& =\left[\left(a_{1}, a_{5}, a_{2}, a_{3}\right),\left(a_{1}, a_{5}, a_{3}, a_{4}\right)\right] .
\end{aligned}
$$

If $n=2$ and $p<q$, then $q=2 n+1=5$ and we can use the equality $[\sigma, \tau]^{-1}=[\tau, \sigma]$. In all cases $a_{1}^{\sigma}=a_{1}^{\tau}$ and if $q \neq 5$, also $a_{1}^{\sigma \sigma} \notin \operatorname{supp} \tau$.

Let $n>2$. The proof is divided into 3 cases.

Case 1: Suppose $q \leq 2 n$. Let $p_{1}=p-2, q_{1}=q-1$, and $n_{1}=n-1$. Then $p_{1}+q_{1}=p-2+q-1 \geq 3 n_{1}+2$ and $p_{1}, q_{1} \leq 2 n_{1}+1$. By the inductive hypothesis there exist a $p_{1}$-cycle $\sigma$, a $q_{1}$-cycle $\tau$, and $a \in \operatorname{supp} \sigma$ such that $[\sigma, \tau]$ is a $\left(2 n_{1}+1\right)$ cycle, $\operatorname{supp} \sigma \cup \operatorname{supp} \tau=\operatorname{supp}[\sigma, \tau]$, and $a^{\sigma}=a^{\tau}$. Let $x, y \notin \operatorname{supp} \sigma \cup \operatorname{supp} \tau, \widetilde{\sigma}=$ $\varphi\left(\sigma ; a^{\sigma}, x, y\right)$, and $\widetilde{\tau}=\varphi\left(\tau ; a^{\tau}, y\right)$. Then $\widetilde{\sigma}$ is a $p$-cycle, $\widetilde{\tau}$ is a $q$-cycle, $a^{\widetilde{\sigma}}=a^{\sigma}=a^{\tau}=$ $a^{\widetilde{\tau}}, a^{\widetilde{\sigma} \widetilde{\sigma}}=x \notin \operatorname{supp} \widetilde{\tau}$, and by Lemma $2.4,[\widetilde{\sigma}, \widetilde{\tau}]$ is a $(2 n+1)$-cycle and supp $\widetilde{\sigma} \cup \operatorname{supp} \widetilde{\tau}=$ $\operatorname{supp}[\widetilde{\sigma}, \widetilde{\tau}]$.

Case 2: Suppose $q=2 n+1$ and $p \neq 2 n+1$. This case follows from the previous case and equality $[\sigma, \tau]^{-1}=[\tau, \sigma]$.

Case 3: Suppose $p=q=2 n+1$. By the inductive hypothesis there exist $(2 n-1)$ cycles $\sigma, \tau$, and $a \in \operatorname{supp} \sigma$ such that $[\sigma, \tau]$ is a $(2 n-1)$-cycle, $\operatorname{supp} \sigma=\operatorname{supp} \tau=$ $\operatorname{supp}[\sigma, \tau]$, and $a^{\sigma}=a^{\tau}$. Let $x, y \notin \operatorname{supp} \sigma, \widetilde{\sigma}=\varphi\left(\sigma ; a^{\sigma}, x, y\right)$, and $\widetilde{\tau}=\varphi\left(\tau ; a^{\tau}, y, x\right)$. Then $\widetilde{\sigma}$ and $\widetilde{\tau}$ are $(2 n+1)$-cycles, $a^{\widetilde{\sigma}}=a^{\sigma}=a^{\tau}=a^{\widetilde{\tau}}$, and by Lemma 2.3, $[\widetilde{\sigma}, \widetilde{\tau}]$ is a $(2 n+1)$-cycle and $\operatorname{supp} \widetilde{\sigma}=\operatorname{supp} \widetilde{\tau}=\operatorname{supp}[\widetilde{\sigma}, \widetilde{\tau}]$.

Lemma 2.6. Let $\sigma, \tau$ be permutations and $a, b \in \operatorname{supp} \sigma$ such that $b=a^{\sigma}=a^{\tau}, b^{\sigma} \notin$ $\operatorname{supp} \tau$, and $c \notin \operatorname{supp} \sigma \cup \operatorname{supp} \tau$. Then

$$
[\sigma, \varphi(\tau ; b, c)]=\varepsilon\left([\sigma, \tau] ; b^{\sigma}\right)\left(c, b^{\sigma}\right) .
$$

Proof. Let $\widetilde{\tau}=\varphi(\tau ; b, c)$. By Corollary 2.2, we get $t^{[\sigma, \widetilde{\tau}]}=t^{[\sigma, \tau]}$ for $t \notin\left\{b^{\sigma}, b^{\tau \sigma}, c\right\}$. From

$$
\begin{aligned}
\left(b^{\sigma}\right)^{[\sigma, \widetilde{\tau}]} & =b^{\widetilde{\tau}^{-1} \sigma \widetilde{\tau}}=a^{\sigma \widetilde{\tau}}=b^{\widetilde{\tau}}=c, \\
c^{[\sigma, \widetilde{\tau}]} & =c^{\widetilde{\tau}^{-1} \sigma \widetilde{\tau}}=b^{\sigma \widetilde{\tau}}=b^{\sigma}, \\
\left(b^{\tau \sigma}\right)^{[\sigma, \widetilde{\tau}]} & =\left(b^{\tau}\right)^{\widetilde{\tau}^{-1} \sigma \widetilde{\tau}}=c^{\sigma \widetilde{\tau}}=c^{\widetilde{\tau}}=b^{\tau},
\end{aligned}
$$


and

$$
\begin{aligned}
\left(b^{\tau \sigma}\right)^{[\sigma, \tau]} & =b^{\sigma \tau}=b^{\sigma}, \\
\left(b^{\sigma}\right)^{[\sigma, \tau]} & =b^{\tau^{-1} \sigma \tau}=a^{\sigma \tau}=b^{\tau},
\end{aligned}
$$

it follows $[\sigma, \varphi(\tau ; b, c)]=\varepsilon\left([\sigma, \tau] ; b^{\sigma}\right)\left(c, b^{\sigma}\right)$.

Corollary 2.7. Let $n_{1}, n_{2} \in \mathbb{N}$ and let $\rho$ be a product of two disjoint cycles of lengths $2 n_{1}$ and $2 n_{2}$, respectively. If $p, q \leq 2\left(n_{1}+n_{2}\right)-1$ and $p+q \geq 3\left(n_{1}+n_{2}\right)$ then there exist a p-cycle $\sigma$, a q-cycle $\tau$, and $a \in \operatorname{supp} \sigma$ such that $\rho=[\sigma, \tau], \operatorname{supp} \rho=\operatorname{supp} \sigma \cup \operatorname{supp} \tau$, and $a^{\sigma}=a^{\tau}$.

If $n_{1}=n_{2}=1$ then there exist no cycles $\sigma$ and $\tau$ such that the length of one of them is strictly greater than $2\left(n_{1}+n_{2}\right)-1=3,[\sigma, \tau]$ is a product of two disjoint transpositions, $\operatorname{supp}[\sigma, \tau]=\operatorname{supp} \sigma \cup \operatorname{supp} \tau$, where $a^{\sigma}=a^{\tau}$ for some $a \in \operatorname{supp} \sigma$. That means that in the Corollary in this case the upper bound requirement on the length of the cycles is sharp. If $n_{1}+n_{2} \geq 3$ the upper bound requirement is not sharp (it can be increased to $\left.2\left(n_{1}+n_{2}\right)\right)$ but the bound in the Corollary is in almost all cases sufficient for our purposes. Namely, in the case $n_{1}+n_{2} \geq 4$, we get $2\left(2\left(n_{1}+n_{2}\right)-2\right) \geq 3\left(n_{1}+n_{2}\right)$ and therefore the Corollary provides two cycles whose lengths can be required to be (independently) either odd or even: both odd $\left(p=q=2\left(n_{1}+n_{2}\right)-1\right)$, both even $\left(p=q=2\left(n_{1}+n_{2}\right)-2\right)$, the first even and the second odd $\left(p=2\left(n_{1}+n_{2}\right)-2, q=2\left(n_{1}+n_{2}\right)-1\right)$, the first odd and the second even.

Proof. One may assume that $n_{1} \geq n_{2}$. The proof is by induction on $n_{2}$.

Let $n_{2}=1$. If $n_{1}=1$ then the only possibility for $p$ and $q$ is $p=q=3$. In this case $\left[\left(a_{1}, a_{2}, a_{3}\right),\left(a_{1}, a_{2}, a_{4}\right)\right]=\left(a_{1}, a_{2}\right),\left(a_{3}, a_{4}\right)$. Let $n_{1} \geq 2$. Because $p+(q-1) \geq$ $3\left(n_{1}+1\right)-1=3 n_{1}+2$ and $p, q \leq 2\left(n_{1}+1\right)-1=2 n_{1}+1$, Corollary 2.5 provides a $p$-cycle $\sigma$, a $(q-1)$-cycle $\tau$, and $a \in \operatorname{supp} \sigma$ such that $[\sigma, \tau]$ is a $\left(2 n_{1}+1\right)$-cycle, $\operatorname{supp} \sigma \cup \operatorname{supp} \tau=\operatorname{supp}[\sigma, \tau], a^{\sigma}=a^{\tau}$, and $a^{\sigma \sigma} \notin \operatorname{supp} \tau$. Let $c \notin \operatorname{supp} \sigma \cup \operatorname{supp} \tau$ and $\widetilde{\tau}=\varphi\left(\tau ; a^{\tau}, c\right)$. Then $\widetilde{\tau}$ is a $q$-cycle, $a^{\sigma}=a^{\tau}=a^{\widetilde{\tau}}$, and by Lemma 2.6, $[\sigma, \widetilde{\tau}]=$ $\varepsilon\left([\sigma, \tau] ; a^{\sigma \sigma}\right)\left(a^{\sigma \sigma}, c\right)$ and $\operatorname{supp} \sigma \cup \operatorname{supp} \widetilde{\tau}=\operatorname{supp}[\sigma, \widetilde{\tau}]$. Note that $a^{\sigma \widetilde{\tau} \sigma}=c$ is in the support of the 2-cycle.

For the proof by induction, suppose that for all $n<n_{2}$ the assumptions $p, q \leq 2\left(n_{1}+\right.$ $n)-1$ and $p+q \geq 3\left(n_{1}+n\right)$ guarantee the existence of a $p$-cycle $\sigma$, a $q$-cycle $\tau$, and $a \in \operatorname{supp} \sigma$ such that the following hold: $[\sigma, \tau]$ is a product of two disjoint cycles of lengths $2 n_{1}$ and $2 n, \operatorname{supp}[\sigma, \tau]=\operatorname{supp} \sigma \cup \operatorname{supp} \tau, a^{\sigma}=a^{\tau}$, and $a^{\sigma \tau \sigma}$ is in the support of the $2 m$-cycle in the cycle decomposition of $[\sigma, \tau]$.

We prove that the same holds for $n=n_{2}$. The proof is divided into 3 cases.

Case 1: Let $q<2\left(n_{1}+n_{2}\right)-1$. Define $\widetilde{p}=p-2, \widetilde{q}=q-1$, and $m=n_{2}-1$. Because $\widetilde{p}+\widetilde{q} \geq 3\left(n_{1}+m\right)$ and $\widetilde{p}, \widetilde{q} \leq 2\left(n_{1}+m\right)-1$, the inductive hypothesis yields a $\widetilde{p}$-cycle $\sigma$, a $\widetilde{q}$-cycle $\tau$, and $a \in \operatorname{supp} \sigma$ such that $[\sigma, \tau]=\rho_{1} \rho_{2}$, where supp $\rho_{1} \cap \operatorname{supp} \rho_{2}=\emptyset, \rho_{1}$ is a $2 n_{1}$-cycle, $\rho_{2}$ is a $2 m$-cycle, $a^{\sigma}=a^{\tau}$, and $a^{\sigma \tau \sigma} \in \operatorname{supp} \rho_{2}$. Let $x, y \notin \operatorname{supp} \sigma \cup \operatorname{supp} \tau$, $\widetilde{\sigma}=\varphi\left(\sigma ; a^{\sigma}, x, y\right)$, and $\widetilde{\tau}=\varphi\left(\tau ; a^{\tau}, y\right)$. Then $\widetilde{\sigma}$ is a $p$-cycle, $\widetilde{\tau}$ is a $q$-cycle, $a^{\widetilde{\sigma}}=$ $a^{\sigma}=a^{\tau}=a^{\widetilde{\tau}}$, and by Lemma 2.4, $[\widetilde{\sigma}, \widetilde{\tau}]=\varphi\left(\rho_{1} \rho_{2} ; a^{\sigma \sigma}, x, y\right)=\rho_{1} \varphi\left(\rho_{2} ; a^{\sigma \sigma}, x, y\right)$ and $a^{\widetilde{\sigma} \widetilde{\sigma}}=a^{\sigma \sigma} \in \operatorname{supp} \varphi\left(\rho_{2} ; a^{\sigma \sigma}, x, y\right)$.

Case 2: Let $p \neq 2\left(n_{1}+n_{2}\right)-1$ and $q=2\left(n_{1}+n_{2}\right)-1$. This case follows from the previous case and the equality $[\sigma, \tau]^{-1}=[\tau, \sigma]$. 
Case 3: Let $p=q=2\left(n_{1}+n_{2}\right)-1$. Define $\tilde{p}=\widetilde{q}=2\left(n_{1}+n_{2}\right)-3$ and $m=n_{2}-1$. From $\tilde{p}, \widetilde{q} \leq 2\left(n_{1}+m\right)-1$ and $n_{1}>1$ we get $\tilde{p}+\widetilde{q} \geq 3\left(n_{1}+m\right)$. By the inductive hypothesis there exist $\widetilde{p}$-cycles $\sigma, \tau$, and $a \in \operatorname{supp} \sigma$ such that $[\sigma, \tau]=\rho_{1} \rho_{2}$, where $\operatorname{supp} \rho_{1} \cap \operatorname{supp} \rho_{2}=\emptyset, \rho_{1}$ is a $2 n_{1}$-cycle, $\rho_{2}$ is a $2 m$-cycle, $a^{\sigma}=a^{\tau}$, and $a^{\sigma \tau \sigma} \in \operatorname{supp} \rho_{2}$. Let $x, y \notin \operatorname{supp} \sigma \cup \operatorname{supp} \tau, \widetilde{\sigma}=\varphi\left(\sigma ; a^{\sigma}, x, y\right)$, and $\widetilde{\tau}=\varphi\left(\tau ; a^{\tau}, y, x\right)$. Then $\widetilde{\sigma}$ and $\widetilde{\tau}$ are $p$-cycles, $a^{\widetilde{\sigma}}=a^{\sigma}=a^{\tau}=a^{\widetilde{\tau}}$, and by Lemma 2.3, $[\widetilde{\sigma}, \widetilde{\tau}]=\varphi\left(\rho_{1} \rho_{2} ; a^{\sigma \tau \sigma}, x, y\right)=$ $\rho_{1} \varphi\left(\rho_{2} ; a^{\sigma \tau \sigma}, x, y\right)$ and $a^{\widetilde{\sigma} \widetilde{\sigma}}=a^{\sigma \sigma} \in \operatorname{supp} \varphi\left(\rho_{2} ; a^{\sigma \tau \sigma}, x, y\right)$.

Lemma 2.8. Let $\sigma, \tau$ be permutations and $a, b \in \operatorname{supp} \sigma$ such that $b=a^{\sigma}=a^{\tau}$, and $x, y, z \notin \operatorname{supp} \sigma \cup \operatorname{supp} \tau$. Then

$$
[\varphi(\sigma ; b, x, y, z), \varphi(\tau ; b, y, z)]=[\sigma, \tau](x, y, z) .
$$

Proof. Let $\widetilde{\sigma}=\varphi(\sigma ; b, x, y, z)$ and $\widetilde{\tau}=\varphi(\tau ; b, y, z)$. By Corollary 2.2, we have $t^{[\tilde{\sigma}, \widetilde{\tau}]}=$ $t^{[\sigma, \tau]}$ for $t \notin\left\{b^{\sigma}, b^{\tau \sigma}, x, y, z\right\}$. As

$$
\begin{aligned}
\left(b^{\sigma}\right)^{[\widetilde{\sigma}, \widetilde{\tau}]} & =z^{\widetilde{\tau}^{-1} \widetilde{\sigma} \widetilde{\tau}}=y^{\widetilde{\sigma} \widetilde{\tau}}=z^{\widetilde{\tau}}=b^{\tau}=\left(a^{\sigma}\right)^{\tau}=\left(b^{\tau^{-1}}\right)^{\sigma \tau}=\left(b^{\sigma}\right)^{[\sigma, \tau]}, \\
\left(b^{\tau \sigma}\right)^{[\widetilde{\sigma}, \widetilde{\tau}]} & =\left(b^{\tau}\right)^{\widetilde{\tau}^{-1} \widetilde{\sigma} \widetilde{\tau}}=z^{\widetilde{\sigma} \widetilde{\tau}}=b^{\sigma \widetilde{\tau}}=b^{\sigma \tau}=\left(b^{\tau \sigma}\right)^{[\sigma, \tau]}, \\
x^{[\widetilde{\sigma}, \widetilde{\tau}]} & =b^{\widetilde{\tau}^{-1} \widetilde{\sigma} \widetilde{\tau}}=a^{\widetilde{\sigma} \widetilde{\tau}}=b^{\widetilde{\tau}}=y, \\
y^{[\widetilde{\sigma}, \widetilde{\tau}]} & =x^{\widetilde{\tau}^{-1} \widetilde{\sigma} \widetilde{\tau}}=x^{\widetilde{\sigma} \widetilde{\tau}}=y^{\widetilde{\tau}}=z, \\
z^{[\widetilde{\sigma}, \widetilde{\tau}]} & =y^{\widetilde{\tau}^{-1} \widetilde{\sigma} \widetilde{\tau}}=b^{\widetilde{\sigma} \widetilde{\tau}}=x^{\widetilde{\tau}}=x,
\end{aligned}
$$

we have $[\widetilde{\sigma}, \widetilde{\tau}]=[\sigma, \tau](x, y, z)$.

Lemma 2.9. Let $\sigma_{1}, \sigma_{2}, \tau_{1}, \tau_{2}$ be cycles such that $\left(\operatorname{supp} \sigma_{1} \cup \operatorname{supp} \tau_{1}\right) \cap\left(\operatorname{supp} \sigma_{2} \cup\right.$ $\left.\operatorname{supp} \tau_{2}\right)=\emptyset$. Suppose there exist $a \in \operatorname{supp} \sigma_{1}$ and $b \in \operatorname{supp} \sigma_{2}$ such that $a^{\sigma_{1}}=a^{\tau_{1}}$ and $b^{\sigma_{2}}=b^{\tau_{2}}$. Then $\left[\psi\left(\sigma_{1}, \sigma_{2} ; a^{\sigma_{1}}, b^{\sigma_{2}}\right), \psi\left(\tau_{1}, \tau_{2} ; a^{\tau_{1}}, b^{\tau_{2}}\right)\right]=\left[\sigma_{1}, \tau_{1}\right]\left[\sigma_{2}, \tau_{2}\right]$.

Proof. Let $\sigma=\psi\left(\sigma_{1}, \sigma_{2} ; a^{\sigma_{1}}, b^{\sigma_{2}}\right)$ and $\tau=\psi\left(\tau_{1}, \tau_{2} ; a^{\tau_{1}}, b^{\tau_{2}}\right)$. Set $c=a^{\sigma_{1}}=a^{\tau_{1}}$ and $d=b^{\sigma_{2}}=b^{\tau_{2}}$. From Corollary 2.2 and equalities $\sigma=\varphi\left(\sigma_{1} ; c, b^{\sigma_{2}^{2}}, \ldots, b, b^{\sigma_{2}}\right)$ and $\tau=\varphi\left(\tau_{1} ; c, b^{\tau_{2}^{2}}, \ldots, b, b^{\tau_{2}}\right)$, we get $t^{[\sigma, \tau]}=t^{\left[\sigma_{1}, \tau_{1}\right]}=t^{\left[\sigma_{1}, \tau_{1}\right]\left[\sigma_{2}, \tau_{2}\right]}$ for $t \notin\left\{c^{\sigma_{1}}, c^{\tau_{1} \sigma_{1}}\right\} \cup$ $\operatorname{supp} \sigma_{2} \cup \operatorname{supp} \tau_{2}$. From Corollary 2.2 and equalities $\sigma=\varphi\left(\sigma_{2} ; d, a^{\sigma_{1}^{2}}, \ldots, a, a^{\sigma_{1}}\right)$ and $\tau=\varphi\left(\tau_{2} ; d, a^{\tau_{1}^{2}}, \ldots, a, a^{\tau_{1}}\right)$, we get $t^{[\sigma, \tau]}=t^{\left[\sigma_{2}, \tau_{2}\right]}=t^{\left[\sigma_{1}, \tau_{1}\right]\left[\sigma_{2}, \tau_{2}\right]}$ for $t \notin\left\{d^{\sigma_{2}}, d^{\tau_{2} \sigma_{1}}\right\} \cup$ $\operatorname{supp} \sigma_{2} \cup \operatorname{supp} \tau_{2}$. Therefore $t^{[\sigma, \tau]}=t^{\left[\sigma_{1}, \tau_{1}\right]\left[\sigma_{2}, \tau_{2}\right]}$ for $t \notin\left\{c^{\sigma_{1}}, c^{\tau_{1} \sigma_{1}}, d^{\sigma_{2}}, d^{\tau_{2} \sigma_{1}}\right\}$. From

$$
\begin{aligned}
\left(c^{\sigma_{1}}\right)^{[\sigma, \tau]} & =d^{\tau^{-1} \sigma \tau}=b^{\sigma \tau}=d^{\tau}=c^{\tau_{1}}=a^{\sigma_{1} \tau_{1}}=c^{\tau_{1}{ }^{-1} \sigma_{1} \tau_{1}}=\left(c^{\sigma_{1}}\right)^{\left[\sigma_{1}, \tau_{1}\right]}, \\
\left(c^{\tau_{1} \sigma_{1}}\right)^{[\sigma, \tau]} & =\left(c^{\tau_{1}}\right)^{\tau^{-1} \sigma \tau}=d^{\sigma \tau}=\left(c^{\sigma_{1}}\right)^{\tau}=c^{\sigma_{1} \tau_{1}}=\left(c^{\tau_{1} \sigma_{1}}\right)^{\left[\sigma_{1}, \tau_{1}\right]}, \\
\left(d^{\sigma_{2}}\right)^{[\sigma, \tau]} & =c^{\tau^{-1} \sigma \tau}=a^{\sigma \tau}=c^{\tau}=d^{\tau_{2}}=b^{\sigma_{2} \tau_{2}}=d^{\tau_{2}{ }^{-1} \sigma_{2} \tau_{2}}=\left(d^{\sigma_{2}}\right)^{\left[\sigma_{2}, \tau_{2}\right]}, \\
\left(d^{\tau_{2} \sigma_{2}}\right)^{[\sigma, \tau]} & =\left(d^{\tau_{2}}\right)^{\tau^{-2} \sigma \tau}=c^{\sigma \tau}=\left(d^{\sigma_{2}}\right)^{\tau}=d^{\sigma_{2} \tau_{2}}=\left(d^{\tau_{2} \sigma_{2}}\right)^{\left[\sigma_{2}, \tau_{2}\right]},
\end{aligned}
$$

we get $[\sigma, \tau]=\left[\sigma_{1}, \tau_{1}\right]\left[\sigma_{2}, \tau_{2}\right]$.

Theorem 2.10. Let $\rho \in A_{n}$. If $n \geq 5$ or $\rho$ is not a 3-cycle then $\rho$ is a commutator of two cycles of $A_{n}$. 
Proof. If $\rho=\left(a_{1}, a_{2}, a_{3}\right)$ is a 3-cycle then $n \geq 5$ and $\rho=\left[\left(a_{1}, a_{3}, x\right),\left(a_{1}, a_{2}, y\right)\right]$ for some $x, y \notin \operatorname{supp} \rho$.

Suppose that $\rho$ is not a 3-cycle. We show that there exist cycles $\sigma$ and $\tau$ of odd lengths and $a \in \operatorname{supp} \sigma$ such that $\rho=[\sigma, \tau], \operatorname{supp} \rho=\operatorname{supp} \sigma \cup \operatorname{supp} \tau$, and $a^{\sigma}=a^{\tau}$. The proof is by induction on the number of cycles in the cycle decomposition of $\rho$, which we denote by $c(\rho)$.

If $c(\rho)=1, \rho$ is a cycle of odd length $l \geq 5$. The statement follows from Corollary 2.5.

If $c(\rho)=2$, then let $\rho=\rho_{1} \rho_{2}$, where $\rho_{1}$ and $\rho_{2}$ are disjoint cycles. The lengths of these cycles are of the same parity. If the lengths are even, the statement follows from Corollary 2.7. In the case of odd lengths, 3 cases are considered.

Case 1: Suppose both lengths are 3. Then $\left[\left(a_{1}, a_{2}, a_{6}, a_{5}, a_{3}\right),\left(a_{1}, a_{2}, a_{4}, a_{6}, a_{5}\right)\right]=$ $\left(a_{1}, a_{2}, a_{3}\right)\left(a_{4}, a_{5}, a_{6}\right)$.

Case 2: Suppose exactly one of the lengths is 3 . One may assume $\rho_{2}=(x, y, z)$ is the 3 -cycle. Let $\rho_{1}$ be a cycle of length $2 l+1$, where $l \geq 2$. By Corollary 2.5 , there exist a $2 l$-cycle $\sigma$, a $(2 l+1)$-cycle $\tau$, and $a \in \operatorname{supp} \sigma$ such that $\rho_{1}=[\sigma, \tau], \operatorname{supp} \rho_{1}=\operatorname{supp} \sigma \cup$ $\operatorname{supp} \tau$, and $a^{\sigma}=a^{\tau}$. By Lemma 2.8, we have $\rho=\left[\varphi\left(\sigma ; a^{\sigma}, x, y, z\right), \varphi\left(\tau ; a^{\tau}, y, z\right)\right]$, where $\varphi\left(\sigma ; a^{\sigma}, x, y, z\right)$ and $\varphi\left(\tau ; a^{\tau}, y, z\right)$ are $(2 l+3)$-cycles.

Case 3: Suppose both lengths are greater than 3. Let $\rho_{i}$ be a cycle of length $2 l_{i}+1$, $l_{i} \geq 2$. By Corollary 2.5, there exist $\left(2 l_{1}+1\right)$-cycles $\sigma_{1}, \tau_{1},\left(2 l_{2}\right)$-cycles $\sigma_{2}, \tau_{2}, a_{1} \in$ $\operatorname{supp} \sigma_{1}$, and $a_{2} \in \operatorname{supp} \sigma_{2}$ such that $\rho_{i}=\left[\sigma_{i}, \tau_{i}\right], \operatorname{supp} \rho_{i}=\operatorname{supp} \sigma_{i} \cup \operatorname{supp} \tau_{i}$, and $a_{i}^{\sigma_{i}}=a_{i}^{\tau_{i}}$. Then $\psi\left(\sigma_{1}, \sigma_{2} ; a_{1}^{\sigma_{1}}, a_{2}^{\sigma_{2}}\right)$ and $\psi\left(\tau_{1}, \tau_{2} ; a_{1}^{\tau_{1}}, a_{2}^{\tau_{2}}\right)$ are $\left(2\left(l_{1}+l_{2}\right)+1\right)$-cycles and by Lemma $2.9, \rho=\left[\psi\left(\sigma_{1}, \sigma_{2} ; a_{1}^{\sigma_{1}}, a_{2}^{\sigma_{2}}\right), \psi\left(\tau_{1}, \tau_{2} ; a_{1}^{\tau_{1}}, a_{2}^{\tau_{2}}\right)\right]$.

If $c(\rho) \geq 3$, the following 4 cases are considered.

Case 1: Suppose $\rho=\rho_{1} \rho_{2}$, where $\rho_{2}$ is a $(2 l+1)$-cycle, $l \geq 2$, and $\operatorname{supp} \rho_{1} \cap \operatorname{supp} \rho_{2}=$ $\emptyset$. By Corollary 2.5, there exist $(2 l)$-cycles $\sigma_{2}, \tau_{2}$ and $b \in \operatorname{supp} \sigma_{2}$, such that $\rho_{2}=\left[\sigma_{2}, \tau_{2}\right]$, $\operatorname{supp} \rho_{2}=\operatorname{supp} \sigma_{2} \cup \operatorname{supp} \tau_{2}$, and $b^{\sigma_{2}}=b^{\tau_{2}}$. Because $2 \leq c\left(\rho_{1}\right) \leq c(\rho)-1$, the inductive hypothesis yields cycles $\sigma_{1}, \tau_{1}$ of odd lengths, as well as $a \in \operatorname{supp} \sigma_{1}$, such that $\rho_{1}=\left[\sigma_{1}, \tau_{1}\right], \operatorname{supp} \rho_{1}=\operatorname{supp} \sigma_{1} \cup \operatorname{supp} \tau_{1}$, and $a^{\sigma_{1}}=a^{\tau_{1}}$. By Lemma 2.9, we have $\rho=$ $\left[\psi\left(\sigma_{1}, \sigma_{2} ; a^{\sigma_{1}}, b^{\sigma_{2}}\right), \psi\left(\tau_{1}, \tau_{2} ; a^{\tau_{1}}, b^{\tau_{2}}\right)\right]$, where $\psi\left(\sigma_{1}, \sigma_{2} ; a^{\sigma_{1}}, b^{\sigma_{2}}\right)$ and $\psi\left(\tau_{1}, \tau_{2} ; a^{\tau_{1}}, b^{\tau_{2}}\right)$ are cycles of odd lengths.

Case 2: Suppose $\rho=\rho_{1} \rho_{2}$, where $\rho_{2}=\left(a_{1}, a_{2}, a_{3}\right)\left(a_{4}, a_{5}, a_{6}\right)$ and $\operatorname{supp} \rho_{1} \cap \operatorname{supp} \rho_{2}$ $=\emptyset$. If $\rho_{1}=\left(a_{7}, a_{8}, a_{9}\right)$ then $\rho=\left[\left(a_{1}, a_{2}, a_{7}, a_{8}, a_{9}, a_{4}, a_{5}, a_{3}, a_{6}\right),\left(a_{1}, a_{2}, a_{8}, a_{9}, a_{5}\right.\right.$, $\left.\left.a_{3}, a_{4}\right)\right]$. If $\rho_{1}$ is not a 3-cycle, the inductive hypothesis yields cycles $\sigma_{1}, \tau_{1}$ of odd lengths, as well as $a \in \operatorname{supp} \sigma_{1}$, such that $\rho_{1}=\left[\sigma_{1}, \tau_{1}\right], \operatorname{supp} \rho_{1}=\operatorname{supp} \sigma_{1} \cup \operatorname{supp} \tau_{1}$, and $a^{\sigma_{1}}=a^{\tau_{1}}=b$. Then $\sigma=\varphi\left(\varphi\left(\sigma_{1} ; b, a_{1}, a_{2}, a_{3}\right) ; b, a_{4}, a_{5}, a_{6}\right)$ and $\tau=\varphi\left(\varphi\left(\tau_{1} ; b, a_{2}, a_{3}\right)\right.$; $\left.b, a_{5}, a_{6}\right)$ are cycles of odd lengths and, using Lemma 2.8 twice, we get $\rho=[\sigma, \tau]$.

Case 3: Suppose $\rho=\rho_{1} \rho_{2}$, where $\rho_{2}$ is a disjoint product of cycles of lengths $2 l_{1}$ and $2 l_{2}$, such that $l_{1}+l_{2} \geq 3$, and supp $\rho_{1} \cap \operatorname{supp} \rho_{2}=\emptyset$.

If $\rho_{1}=\left(a_{1}, a_{2}, a_{3}\right)$ then by Corollary 2.7 , there exist a $\left(2\left(l_{1}+l_{2}\right)-2\right)$-cycle $\sigma_{2}$, a $\left(2\left(l_{1}+l_{2}\right)-1\right)$-cycle $\tau_{2}$, and $a \in \operatorname{supp} \sigma_{2}$, such that $\rho_{2}=\left[\sigma_{2}, \tau_{2}\right], \operatorname{supp} \rho_{2}=\operatorname{supp} \sigma_{2} \cup$ $\operatorname{supp} \tau_{2}$, and $a^{\sigma_{2}}=a^{\tau_{2}}=b$. Then $\sigma=\varphi\left(\sigma_{2} ; b, a_{1}, a_{2}, a_{3}\right)$ and $\tau=\varphi\left(\tau_{2} ; b, a_{2}, a_{3}\right)$ are $\left(2\left(l_{1}+l_{2}\right)+1\right)$-cycles and by Lemma 2.8 , we get $\rho=[\sigma, \tau]$.

If $\rho_{1}$ is not a 3-cycle then by the inductive hypothesis there exist cycles $\sigma_{1}, \tau_{1}$ of odd lengths and $a \in \operatorname{supp} \sigma_{1}$, such that $\rho_{1}=\left[\sigma_{1}, \tau_{1}\right], \operatorname{supp} \rho_{1}=\operatorname{supp} \sigma_{1} \cup \operatorname{supp} \tau_{1}$, and $a^{\sigma_{1}}=$ $a^{\tau_{1}}$. If $l_{1}+l_{2}=3$ then $\rho_{2}=\left(a_{1}, a_{2}, a_{3}, a_{4}\right)\left(a_{5}, a_{6}\right)$ and for $\sigma_{2}=\left(a_{1}, a_{5}, a_{2}, a_{4}, a_{6}, a_{3}\right)$ and $\tau_{2}=\left(a_{1}, a_{5}, a_{3}, a_{4}\right)$ we get $\rho_{2}=\left[\sigma_{2}, \tau_{2}\right]$ and for $b=a_{1}$ we get $b^{\sigma_{1}}=b^{\tau_{1}}$. If $l_{1}+l_{2}>$ 3 Corollary 2.7 provides $\left(2\left(l_{1}+l_{2}\right)-2\right)$-cycles $\sigma_{2}$ and $\tau_{2}$, as well as $b \in \operatorname{supp} \sigma_{2}$, such that 
$\rho_{2}=\left[\sigma_{2}, \tau_{2}\right], \operatorname{supp} \rho_{2}=\operatorname{supp} \sigma_{2} \cup \operatorname{supp} \tau_{2}$, and $b^{\sigma_{2}}=b^{\tau_{2}}$. Then $\sigma=\psi\left(\sigma_{1}, \sigma_{2} ; a^{\sigma_{1}}, b^{\sigma_{2}}\right)$ and $\tau=\psi\left(\tau_{1}, \tau_{2} ; a^{\tau_{1}}, b^{\tau_{2}}\right)$ are cycles of odd length and by Lemma 2.9, we get $\rho=[\sigma, \tau]$.

Case 4: Suppose $\rho$ is a disjoint product of transpositions and at most one 3-cycle. If there are at most four transpositions in the cycle decomposition of $\rho$ we have 3 possibilities:

$$
\begin{aligned}
& {\left[\left(a_{1}, a_{3}, a_{5}, a_{6}, a_{2}, a_{4}, a_{7}\right),\left(a_{1}, a_{3}, a_{6}, a_{4}, a_{7}, a_{2}, a_{5}\right)\right]=\left(a_{1}, a_{2}\right)\left(a_{3}, a_{4}\right)\left(a_{5}, a_{6}, a_{7}\right),} \\
& {\left[\left(a_{1}, a_{2}, a_{4}, a_{8}, a_{6}, a_{3}, a_{5}\right),\left(a_{1}, a_{2}, a_{3}, a_{8}, a_{4}, a_{6}, a_{7}\right)\right]=\left(a_{1}, a_{2}\right)\left(a_{3}, a_{4}\right)\left(a_{5}, a_{6}\right)\left(a_{7}, a_{8}\right),} \\
& {\left[\left(a_{1}, a_{2}, a_{5}, a_{3}, a_{4}, a_{9}, a_{10}, a_{7}, a_{11}\right),\left(a_{1}, a_{2}, a_{6}, a_{3}, a_{4}, a_{10}, a_{7}, a_{9}, a_{8}\right)\right]=} \\
& \quad=\left(a_{1}, a_{2}\right)\left(a_{3}, a_{4}\right)\left(a_{5}, a_{6}\right)\left(a_{7}, a_{8}\right)\left(a_{9}, a_{10}, a_{11}\right) .
\end{aligned}
$$

Otherwise $\rho=\rho_{1} \rho_{2}$, where $\rho_{2}=\left(a_{1}, a_{2}\right)\left(a_{3}, a_{4}\right)\left(a_{5}, a_{6}\right)\left(a_{7}, a_{8}\right), 2 \leq c\left(\rho_{1}\right)<c(\rho)$, and supp $\rho_{1} \cap \operatorname{supp} \rho_{2}=\emptyset$. By the inductive hypothesis there exist cycles $\sigma_{1}, \tau_{1}$ of odd lengths and $a \in \operatorname{supp} \sigma_{1}$, such that $\rho_{1}=\left[\sigma_{1}, \tau_{1}\right]$, supp $\rho_{1}=\operatorname{supp} \sigma_{1} \cup \operatorname{supp} \tau_{1}$, and $a^{\sigma_{1}}=a^{\tau_{1}}$. For $\sigma_{2}=\left(a_{1}, a_{8}, a_{3}, a_{2}, a_{4}, a_{6}, a_{7}, a_{5}\right)$ and $\tau_{2}=\left(a_{1}, a_{8}, a_{4}, a_{3}, a_{5}, a_{6}\right)$ we have $\rho_{2}=\left[\sigma_{2}, \tau_{2}\right]$. Then $\sigma=\psi\left(\sigma_{1}, \sigma_{2} ; a^{\sigma_{1}}, a_{1}^{\sigma_{2}}\right)$ and $\tau=\psi\left(\tau_{1}, \tau_{2} ; a^{\tau_{1}}, a_{1}^{\tau_{2}}\right)$ are cycles of odd lengths and by Lemma 2.9, we get $\rho=[\sigma, \tau]$.

\section{Cycles as commutators of cycles}

From the previous section we know that a $(2 n+1)$-cycle is a commutator of a $p$-cycle and a $q$-cycle if $p+q \geq 3 n+2$ (and $p, q \leq 2 n+1$ ). But this sufficient condition is not necessary. Note that in the previous section we were interested in pairs of cycles $\sigma$ and $\tau$, for which there exists $a \in \operatorname{supp} \sigma$ such that $a^{\sigma}=a^{\tau}$. We needed that for "concatenation" of cycles in Lemma 2.9. With that assumption withdrawn, the result is obtained by using a more stringent hypothesis as shown in the next corollary.

Lemma 3.1. Let $\sigma, \tau$ be permutations, $x, y \notin \operatorname{supp} \sigma \cup \operatorname{supp} \tau, a_{1}, a_{2} \in \operatorname{supp} \sigma \cap \operatorname{supp} \tau$, $b \in \operatorname{supp} \sigma-\operatorname{supp} \tau$, and $c \in \operatorname{supp} \tau-\operatorname{supp} \sigma$, such that $a_{1}^{\sigma}=b, b^{\sigma}=a_{2}, a_{1}^{\tau}=c$, and $c^{\tau}=a_{2}$. Then

$$
[\varphi(\sigma ; b, c, x), \varphi(\tau ; c, y)]=\varphi([\sigma, \tau] ; c, y, x)
$$

Proof. Let $\widetilde{\sigma}=\varphi(\sigma ; b, c, x)$ and $\widetilde{\tau}=\varphi(\tau ; c, y)$. If $t \notin\left\{x, a_{2}, c\right\}$ then $t^{\sigma^{-1}}=t^{\widetilde{\sigma}^{-1}}$. If $t \notin\left\{y, a_{2}^{\sigma}\right\}$ then $t^{\sigma^{-1}} \notin\left\{y, a_{2}\right\}$ and $t^{\sigma^{-1}} \tau^{-1}=t^{\sigma^{-1}} \widetilde{\tau}^{-1}$. If $t \notin\left\{x, a_{2}^{\sigma}, a_{2}\right\}$ then $t^{\sigma^{-1} \tau^{-1}} \notin\{x, c, b\}$ and $t^{\sigma^{-1} \tau^{-1} \sigma}=t^{\sigma^{-1} \tau^{-1} \widetilde{\sigma}}$. If $t \notin\left\{y, a_{2}^{\sigma}\right\}$ then $t^{\sigma^{-1} \tau^{-1} \sigma} \notin\{y, c\}$ and $t^{\sigma^{-1} \tau^{-1} \sigma \tau}=t^{\sigma^{-1} \tau^{-1} \tilde{\sigma} \widetilde{\tau}}$. Hence for $t \notin\left\{x, y, c, a_{2}, a_{2}^{\sigma}\right\}$ we get $t^{[\sigma, \tau]}=t^{[\widetilde{\sigma}, \widetilde{\tau}]}$. Because

$$
\begin{aligned}
c^{[\sigma, \tau]} & =c^{\tau^{-1} \sigma \tau}=a_{1}^{\sigma \tau}=b^{\tau}=b, \\
c^{[\widetilde{\sigma}, \widetilde{\tau}]} & =b^{\widetilde{\tau}^{-1} \widetilde{\sigma} \widetilde{\tau}}=b^{\widetilde{\sigma} \widetilde{\tau}}=c^{\widetilde{\tau}}=y, \\
y^{[\widetilde{\sigma}, \widetilde{\tau}]} & =y^{\widetilde{\tau}^{-1} \widetilde{\sigma} \widetilde{\tau}}=c^{\widetilde{\sigma} \widetilde{\tau}}=x^{\widetilde{\tau}}=x, \\
x^{[\widetilde{\sigma}, \widetilde{\tau}]} & =c^{\widetilde{\tau}^{-1} \widetilde{\tau} \widetilde{\tau}}=a_{1}^{\widetilde{\sigma} \widetilde{\tau}}=b^{\widetilde{\tau}}=b, \\
a_{2}^{[\widetilde{\sigma}, \widetilde{\tau}]} & =x^{\widetilde{\tau}^{-1} \widetilde{\sigma} \widetilde{\tau}}=x^{\widetilde{\sigma} \widetilde{\tau}}=a_{2}^{\widetilde{\tau}}=a_{2}^{\tau}=b^{\sigma \tau}=b^{\tau^{-1} \sigma \tau}=a_{2}^{[\sigma, \tau]}, \\
\left(a_{2}^{\sigma}\right)^{[\widetilde{\sigma}, \widetilde{\tau}]} & =a_{2}^{\widetilde{\tau}^{-1} \widetilde{\sigma} \widetilde{\tau}}=y^{\widetilde{\sigma} \widetilde{\tau}}=y^{\widetilde{\tau}}=a_{2}=c^{\tau}=c^{\sigma \tau}=a_{2}^{\tau^{-1} \sigma \tau}=\left(a_{2}^{\sigma}\right)^{[\sigma, \tau]},
\end{aligned}
$$

we get $[\widetilde{\sigma}, \widetilde{\tau}]=\varphi([\sigma, \tau] ; c, y, x)$. 
Corollary 3.2. Let $\rho$ be a $(2 n+1)$-cycle and $n \geq 2$. For $p, q \in \mathbb{N}$ such that $p, q \leq 2 n$ and $p+q=3 n+1$, there exist a $p$-cycle $\sigma$ and a q-cycle $\tau$, such that $[\sigma, \tau]=\rho$ and $\operatorname{supp} \rho=\operatorname{supp} \sigma \cup \operatorname{supp} \tau$.

Proof. By induction on $n$ we prove that whenever $p, q \leq 2 n$ and $p+q=3 n+1$, there exist a $p$-cycle $\sigma$, a $q$-cycle $\tau, a_{1}, a_{2} \in \operatorname{supp} \sigma \cap \operatorname{supp} \tau, b \in \operatorname{supp} \sigma-\operatorname{supp} \tau$, and $c \in \operatorname{supp} \tau-\operatorname{supp} \sigma$, such that $a_{1}^{\sigma}=b, b^{\sigma}=a_{2}, a_{1}^{\tau}=c, c^{\tau}=a_{2},[\sigma, \tau]$ is a $(2 n+1)$ cycle, and $\operatorname{supp}[\sigma, \tau]=\operatorname{supp} \sigma \cup \operatorname{supp} \tau$.

Because $[\tau, \sigma]=[\sigma, \tau]^{-1}$ we may assume $p \geq q$.

If $n=2$ then $p=4, q=3$ and we have $\left[\left(a_{1}, b, a_{2}, d\right),\left(a_{1}, c, a_{2}\right)\right]=\left(a_{1}, c, b, d, a_{2}\right)$.

Let $n>2$. For $p, q \leq 2 n$ and $p+q=3 n+1$ we define $\widetilde{p}=p-2$ and $\widetilde{q}=q-1$. Then $\widetilde{p}+\widetilde{q}=3(n-1)+1$ and $\widetilde{p} \leq 2(n-1)$. From $q \leq p$ we get $q \neq 2 n$ and therefore $\widetilde{q} \leq 2(n-1)$. By the inductive hypothesis there exist a $\widetilde{p}$-cycle $\widetilde{\sigma}$, a $\widetilde{q}$-cycle $\widetilde{\tau}$, $a_{1}, a_{2} \in \operatorname{supp} \tilde{\sigma} \cap \operatorname{supp} \widetilde{\tau}, b \in \operatorname{supp} \widetilde{\sigma}-\operatorname{supp} \widetilde{\tau}$, and $c \in \operatorname{supp} \widetilde{\tau}-\operatorname{supp} \widetilde{\sigma}$, such that $a_{1}^{\widetilde{\sigma}}=b$, $b^{\widetilde{\sigma}}=a_{2}, a_{1}^{\widetilde{\tau}}=c, c^{\widetilde{\tau}}=a_{2},[\widetilde{\sigma}, \widetilde{\tau}]$ is a $(2 n-1)$-cycle, and $\operatorname{supp}[\widetilde{\sigma}, \widetilde{\tau}]=\operatorname{supp} \widetilde{\sigma} \cup \operatorname{supp} \tilde{\tau}$. Let $x, y \notin \operatorname{supp} \widetilde{\sigma} \cup \operatorname{supp} \widetilde{\tau}$. Then $\sigma=\varphi(\widetilde{\sigma} ; b, c, x)$ is a $p$-cycle, $\tau=\varphi(\tilde{\tau} ; c, y)$ is a $q$-cycle, $c, a_{2} \in \operatorname{supp} \sigma \cap \operatorname{supp} \tau, x \in \operatorname{supp} \sigma-\operatorname{supp} \tau, y \in \operatorname{supp} \tau-\operatorname{supp} \sigma, c^{\sigma}=x, x^{\sigma}=a_{2}$, $c^{\tau}=y, y^{\tau}=a_{2}$, and by Lemma 3.1, $[\sigma, \tau]$ is a $(2 n+1)$-cycle.

Let $\sigma$ and $\tau$ be permutations. An equivalence relation on the set $\operatorname{supp} \sigma \cap \operatorname{supp} \tau$ is defined in the following way. Elements $a, b \in \operatorname{supp} \sigma \cap \operatorname{supp} \tau$ are equivalent if and only if there exist $a_{0}, \ldots, a_{n} \in \operatorname{supp} \sigma \cap \operatorname{supp} \tau$ and $\rho_{1}, \ldots, \rho_{n} \in\left\{\sigma, \sigma^{-1}, \tau, \tau^{-1}\right\}$, such that $a=a_{0}, b=a_{n}$, and $a_{i}=a_{i-1}^{\rho_{i}}$ for $i=1, \ldots, n$. This is obviously an equivalence relation.

Definition 3.3. Permutations $\sigma$ and $\tau$ are braided if all elements of $\operatorname{supp} \sigma \cap \operatorname{supp} \tau$ are equivalent to each other.

Lemma 3.4. Let $\sigma$ and $\tau$ be cycles such that the commutator $[\sigma, \tau]$ is a cycle and $\operatorname{supp}[\sigma, \tau]$ $=\operatorname{supp} \sigma \cup \operatorname{supp} \tau$. Then $\sigma$ and $\tau$ are braided.

Proof. Let $\rho=[\sigma, \tau]$ and $a_{0} \in \operatorname{supp} \sigma \cap \operatorname{supp} \tau$. For $n \geq 0$ we inductively define $a_{4 n+1}=a_{4 n}^{\sigma^{-1}}, a_{4 n+2}=a_{4 n+1}^{\tau^{-1}}, a_{4 n+3}=a_{4 n+2}^{\sigma}$, and $a_{4 n+4}=a_{4 n+3}^{\tau}$. Let us show that if $a_{4 m}=a_{0}^{\rho^{m}} \in \operatorname{supp} \sigma \cap \operatorname{supp} \tau$, then $a_{4 m}$ is equivalent to $a_{0}$. Let $b_{1}=a_{0}$ and $i_{1}=\max \left\{i \mid i \leq 4 m, a_{i}=a_{0}\right\}$. For $k \geq 1$ and $i_{k}<4 m$ we let $i_{k+1}=\max \left\{i \mid i_{k}<\right.$ $\left.i \leq 4 m, a_{i}=a_{i_{k}+1}\right\}, b_{k+1}=a_{i_{k+1}}$, and $\rho_{k} \in\left\{\sigma, \sigma^{-1}, \tau, \tau^{-1}\right\}$, where $\rho_{k}$ is uniquely defined by $b_{k}^{\rho_{k}}=b_{k+1}$. If we show that $b_{k} \in \operatorname{supp} \sigma \cap \operatorname{supp} \tau$ for all $k$, then by definition, $a_{0}=b_{1}$ is equivalent to $a_{4 m}=b_{l}$. For $1 \leq k<l$ we have $b_{k+1} \in \operatorname{supp} \rho_{k}$. Suppose $b_{k+1} \notin \operatorname{supp} \widetilde{\rho}$, where $\widetilde{\rho}$ is the cycle in $\{\sigma, \tau\}-\left\{\rho_{k}, \rho_{k}^{-1}\right\}$. Because $a_{i_{k}}^{\rho_{k}}=a_{i_{k}+1}$ and $\rho_{k} \neq \widetilde{\rho}^{ \pm 1}$, necessarily also $a_{i_{k}+1}^{\widetilde{\sigma}}=a_{i_{k}+2}$ or $a_{i_{k}+1}^{\widetilde{\sigma}^{-1}}=a_{i_{k}+2}$. Because $a_{i_{k}+2}^{\rho_{k}^{-1}}=a_{i_{k}+3}$ and $a_{i_{k}+1} \notin \operatorname{supp} \widetilde{\sigma}$, we get $a_{i_{k}}=a_{i_{k}+3}$. This contradicts the definition of $i_{k}$. Hence $b_{k} \in \operatorname{supp} \sigma \cap \operatorname{supp} \tau$.

Let $b \in \operatorname{supp} \sigma \cap \operatorname{supp} \tau$. Because $\rho$ is a cycle and $b \in \operatorname{supp} \rho$, there exists $m$ such that $b=a_{0}^{\rho^{m}}$. Thus $b$ is equivalent to $a_{0}$, and hence $\sigma$ and $\tau$ are braided.

Lemma 3.5. Let $\sigma$ and $\tau$ be permutations such that $\operatorname{supp}[\sigma, \tau]=\operatorname{supp} \sigma \cup \operatorname{supp} \tau$. Then $|\operatorname{supp} \sigma-\operatorname{supp} \tau|,|\operatorname{supp} \tau-\operatorname{supp} \sigma| \leq|\operatorname{supp} \sigma \cap \operatorname{supp} \tau|$.

Proof. Suppose there exist $x, y \in \operatorname{supp} \sigma-\operatorname{supp} \tau$, such that $x=y^{\sigma}$. Then $x^{[\sigma, \tau]}=x$, and consequently $x \notin \operatorname{supp}[\sigma, \tau]$, which is a contradiction. Hence the map $(\operatorname{supp} \sigma-$ 
$\operatorname{supp} \tau) \rightarrow(\operatorname{supp} \sigma \cap \operatorname{supp} \tau)$, defined by $x \mapsto x^{\sigma}$, is an injection. Therefore $\mid \operatorname{supp} \sigma-$ $\operatorname{supp} \tau|\leq| \operatorname{supp} \sigma \cap \operatorname{supp} \tau \mid$.

Because $\operatorname{supp}[\tau, \sigma]=\operatorname{supp}[\sigma, \tau]$, the other inequality follows from the above paragraph.

Lemma 3.6. Let $\sigma$ and $\tau$ be cycles such that $[\sigma, \tau]$ is a cycle and $\operatorname{supp}[\sigma, \tau]=\operatorname{supp} \sigma \cup$ $\operatorname{supp} \tau$. Then $|\operatorname{supp} \sigma-\operatorname{supp} \tau|+|\operatorname{supp} \tau-\operatorname{supp} \sigma| \leq|\operatorname{supp} \sigma \cap \operatorname{supp} \tau|+1$.

Proof. Let $k=|\operatorname{supp} \sigma \cap \operatorname{supp} \tau|,|\operatorname{supp} \sigma|=k+p$, and $|\operatorname{supp} \tau|=k+q$. If $p=0$, then by Lemma 3.5 we have

$|\operatorname{supp} \sigma-\operatorname{supp} \tau|+|\operatorname{supp} \tau-\operatorname{supp} \sigma|=|\operatorname{supp} \tau-\operatorname{supp} \sigma|<|\operatorname{supp} \sigma \cap \operatorname{supp} \tau|+1$.

Analogously for $q=0$. Let $p, q>0$. Let $\operatorname{supp} \sigma-\operatorname{supp} \tau=\left\{a_{1}, \ldots, a_{p}\right\}$. Let $m_{i} \in$ $\mathbb{N} \cup\{0\}$ be the largest number such that $a_{i}^{\sigma^{j}} \in \operatorname{supp} \sigma \cap \operatorname{supp} \tau$ for all $j \in\left\{1, \ldots, m_{i}\right\}$. We claim that all $m_{i}$ are positive. Indeed, suppose that there exist $x, y \in \operatorname{supp} \sigma-\operatorname{supp} \tau$, such that $x^{\sigma}=y$. Then $y^{[\sigma, \tau]}=y$ which is a contradiction since $\operatorname{supp} \sigma \subset \operatorname{supp}[\sigma, \tau]$. Hence the set $M_{i}=\left\{a_{i}^{\sigma}, \ldots, a_{i}^{\sigma^{m_{i}}}\right\}$ is nonempty for all $i$. Because $\sigma$ is a cycle and $p>0$, for every $x \in \operatorname{supp} \sigma \cap \operatorname{supp} \tau$ there exists the smallest $i \in \mathbb{N}$ such that $x^{\sigma^{-i}}=a_{k}$ for some $k$, which means that $x \in M_{k}$. Therefore, $(\operatorname{supp} \sigma \cap \operatorname{supp} \tau)=M_{1} \coprod \ldots \coprod M_{p}$. Similarly, $(\operatorname{supp} \sigma \cap \operatorname{supp} \tau)=N_{1} \amalg \ldots \amalg N_{q}$, where $\operatorname{supp} \tau-\operatorname{supp} \sigma=\left\{b_{1}, \ldots, b_{q}\right\}$, $N_{i}=\left\{b_{i}^{\tau}, \ldots, b_{i}^{\tau^{n_{i}}}\right\} \subset \operatorname{supp} \tau \cap \operatorname{supp} \sigma$, and $b_{i}^{\tau^{n_{i}+1}} \notin \operatorname{supp} \sigma$.

By Lemma 3.4, the cycles $\sigma$ and $\tau$ are braided. Hence there exist $i_{2} \in\{2, \ldots, p\}$, $d_{2} \in M_{1}, c_{2} \in M_{i_{2}}$, and $\tau_{2} \in\left\{\tau, \tau^{-1}\right\}$ such that $d_{2}=c_{2}^{\tau_{2}}$. For $j>2$ there exist $i_{j} \in\{2, \ldots, p\}-\left\{i_{2}, \ldots, i_{j-1}\right\}, d_{j} \in M_{1} \cup\left(\cup_{l=2}^{j-1} M_{i_{l}}\right), c_{j} \in M_{i_{j}}$, and $\tau_{j} \in\left\{\tau, \tau^{-1}\right\}$ such that $d_{j}=c_{j}^{\tau_{j}}$. Let us show that for each $i$, the set $\widetilde{N}_{i}=N_{i}-\left\{c_{2}, \ldots, c_{p}\right\}$ is nonempty. By construction, the elements $c_{2}, \ldots, c_{p}$ are different, $d_{j} \neq c_{k}$ for $j \leq k$, and every pair $\left\{c_{j}, d_{j}\right\}$ is a subset of $N_{l}$ for some $l$. Suppose $N_{i} \cap\left\{c_{2}, \ldots, c_{p}\right\}=\left\{c_{k_{1}}, \ldots, c_{k_{r}}\right\}$, where $k_{1}<\ldots<k_{r}$. Then $d_{k_{1}} \in N_{i}$ and $d_{k_{1}} \notin\left\{c_{k_{1}}, \ldots, c_{k_{r}}\right\}$, so $d_{k_{1}} \in \widetilde{N}_{i} \neq \emptyset$. Hence in the union of the $q$ nonempty sets $\widetilde{N}_{1}, \ldots, \widetilde{N}_{q}$ there are exactly $k-(p-1)$ elements. This means that $|\operatorname{supp} \tau-\operatorname{supp} \sigma|=q \leq k-(p-1)=|\operatorname{supp} \sigma \cap \operatorname{supp} \tau|-|\operatorname{supp} \sigma-\operatorname{supp} \tau|+1$.

Theorem 3.7. Let $n \geq 2$ and let $\rho$ be $a(2 n+1)$-cycle. There exist a p-cycle $\sigma$ and $a$ $q$-cycle $\tau$ such that $\rho=[\sigma, \tau]$ and $\operatorname{supp} \rho=\operatorname{supp} \sigma \cup \operatorname{supp} \tau$ if and only if the following three conditions are satisfied (i) $n+1 \leq p$, q, (ii) $2 n+1 \geq p$, q, (iii) $p+q \geq 3 n+1$.

Proof. Suppose there exist a $p$-cycle $\sigma$ and a $q$-cycle $\tau$ such that $\rho=[\sigma, \tau]$ and $\operatorname{supp} \rho=$ $\operatorname{supp} \sigma \cup \operatorname{supp} \tau$. Let $k=|\operatorname{supp} \sigma \cap \operatorname{supp} \tau|, p=k+\widetilde{p}$, and $q=k+\widetilde{q}$. By Lemma 3.5, we have $\widetilde{q} \leq k$, therefore $2 \widetilde{q} \leq k+\widetilde{q}=q \leq 2 n+1$ which implies $\widetilde{q} \leq n$. Then $2 n+1=|\operatorname{supp} \rho|=|\operatorname{supp} \sigma \cup \operatorname{supp} \tau|=p+\widetilde{q} \leq p+n$, hence $n+1 \leq p$. By Lemma 3.6, we have $\widetilde{p}+\widetilde{q} \leq k+1$. Therefore $2 n+1=k+\widetilde{p}+\widetilde{q} \leq 2 k+1$ and $p+q=2 n+1+k \geq 3 n+1$.

If $p+q \geq 3 n+2$ the theorem follows from Corollary 2.5. If $p+q=3 n+1$, the theorem follows from Corollary 3.2.

\section{References}

[1] E. Bertram, Even permutations as a product of two conjugate cycles, J. Combin. Theory 12 (1972), 368-380. 
[2] O. Bonten, Über Kommutatoren in endlichen einfachen Gruppen, Aachener Beiträge zur Math. 7, Verlag der Augustinus-Buchhandlung, Aachen, 1993.

[3] E. W. Ellers, N. Gordeev, On the conjectures of J. Thompson and O. Ore, Trans. Amer. Math. Soc. 350 (1998), no. 9, 3657-3671.

[4] R. Gow, Commutators in the symplectic group, Arch. Math. (Basel) 50 (1988), no. 3, 204-209.

[5] L. C. Kappe, R. S. Morse, On commutators in groups, Groups St. Andrews 2005. Vol. 2, 531558, London Math. Soc. Lecture Note Ser., 340, Cambridge Univ. Press, Cambridge, 2007.

[6] M. W. Liebeck, E. A. O'Brien, A. Shalev, P. H. Tiep, The Ore conjecture, J. Eur. Math. Soc. (JEMS) 12 (2010), no. 4, 939-1008.

[7] G. A. Miller, On the commutators of a given group, Bull. Amer. Math. Soc. 6 (1899) 105-109.

[8] J. Neubüser, H. Pahlings, E. Cleuvers, Each sporadic finasig $G$ has a class $C$ such that $C C=$ G, Abstracts Amer. Math. Soc. 34, 6 (1984).

[9] O. Ore, Some remarks on commutators, Proc. Amer. Math. Soc. 272 (1951), 307-314.

[10] R. C. Thompson, Commutators in the special and general linear groups, Trans. Amer. Math. Soc. 101 (1961), 16-33.

[11] R. C. Thompson, Commutators of matrices with coefficients from the field of two elements, Duke Math. J. 29 (1962), 367-373.

[12] R. C. Thompson, On matrix commutators, Portugal. Math. 21 (1962), 143-153. 